\title{
Asymptotic behavior of regularized scattering phases for long range perturbations
}

\author{
Jean-Marc Bouclet
}

\begin{abstract}
We define scattering phases for Schrödinger operators on $\mathbb{R}^{d}$ as limit of arguments of relative determinants. These phases can be defined for long range perturbations of the Laplacian and therefore they can replace the usual spectral shift function (SSF) of Birman-Krein's theory, which can be defined for only special short range perturbations (relatively trace class perturbations). We prove the existence of asymptotic expansions for these phases, which generalize results on the SSF.
\end{abstract}

\section{Introduction}

\subsection{Assumptions and statement of the problem}

In this article, we will mainly consider perturbations of the Laplacian operator $H_{0}=-\sum_{j=1}^{d} \partial_{j}^{2}$ on $L^{2}\left(\mathbb{R}^{d}\right), d \geq 1$. These perturbations are self-adjoint and defined by

$$
H_{1}=H_{0}+V=-\sum_{1 \leq j, k \leq d} g_{j k}(x) \partial_{j} \partial_{k}+\sum_{j=1}^{d} b_{j}(x) \partial_{j}+c(x)
$$

which means that the perturbation $V$ is a differential operator of order $\leq 2$.

We assume that $H_{1}$ is uniformly elliptic, that is

$$
\sum_{1 \leq j, k \leq d} g_{j k}(x) \xi_{j} \xi_{k} \geq c|\xi|^{2}, \quad \forall x, \xi \in \mathbb{R}^{d}
$$

for some $c>0$; its coefficients are smooth and satisfy for each multiindex $\alpha$

$$
\sum_{j, k}\left|\partial^{\alpha}\left(g_{j k}(x)-\delta_{j k}\right)\right|+\sum_{j}\left|\partial^{\alpha} b_{j}(x)\right|+\left|\partial^{\alpha} c(x)\right| \leq C_{\alpha}\langle x\rangle^{-\rho-|\alpha|},
$$

for all $x \in \mathbb{R}^{d}$; here $\delta_{j k}$ is the usual Kronecker's symbol and $\langle x\rangle=\left(1+|x|^{2}\right)^{1 / 2}$. 
The number $\rho>0$ is a real number, and the condition (1) is a long range condition. Our purpose is to define and study relative scattering determinants

$$
D_{p}(z)=\operatorname{Det}_{p}\left(1+V\left(H_{0}-z\right)^{-1}\right), \quad z \in \mathbb{C} \backslash \mathbb{R}
$$

and the associated phases which are, in a sense, the limits of $\pi^{-1} \arg D_{p}(z)$ when $z$ approaches the real axis. The important point is that we will consider (1), with a general $\rho>0$ in order to relax the usual condition $\rho>d$ needed to use BirmanKrein's theory.

Our definition of $\operatorname{Det}_{p}$ is an extension of the Fredholm determinants defined for compact perturbations of identity which are in $S_{p}$, the Schatten class of order $p$ (see the next subsection for the standard definition of $\operatorname{Det}_{p}$ ). We need such an extension since we want to consider second order perturbations of the Laplacian, for which $V\left(H_{0}-z\right)^{-1}$ is not compact in general.

The organization of this paper is the following: in the very next subsection, we recall some points on perturbations by compact operators in $S_{p}$, especially for $p=1$, with the Birman-Krein's theory of trace class perturbations, and in the next one we review the applications of this theory to differential operators. In section 2, we explain the definitions of $D_{p}(z)$ and of the scattering phase $s_{p}$, and we state our main theorem on the asymptotic behavior of $s_{p}$. In the last section we sketch the proof of this theorem.

\subsection{Compact perturbations. Birman-Krein's and Koplienko's theories}

Let $A_{0}$ be a self-adjoint operator, bounded or not, on a separable Hilbert space. We want to study another self-adjoint operator $A_{1}$, considered as a perturbation of $A_{0}$. If the difference $W:=A_{1}-A_{0}$ is trace class, i.e. is compact and such that its spectrum, $\operatorname{spec}(W)=\left(\mu_{j}\right)_{j \in \mathbb{N}}$, satisfies $\sum_{\mathbb{N}}\left|\mu_{j}\right|<\infty$, Krein has proved that for a wide class of functions $f$, including the Schwartz class $\mathcal{S}(\mathbb{R}), f\left(A_{1}\right)-f\left(A_{0}\right)$ is trace class and there exists a measurable function $s_{1}$ such that

$$
\operatorname{Tr}\left(f\left(A_{1}\right)-f\left(A_{0}\right)\right)=-\int f^{\prime}(\lambda) s_{1}(\lambda) d \lambda, \quad \forall f \in \mathcal{S}(\mathbb{R}) .
$$

This identity defines $s_{1}$ up to a constant term, however, under our assumptions, $s_{1}$ can be chosen in $L^{1}(\mathbb{R})$, and with this condition it is uniquely determined. Further on we shall write $s_{1}(\lambda)=s_{1}\left(A_{0}, A_{1}, \lambda\right)$ to specify the operators (see the next subsection).

This function is usually called the spectral shift function. For references see Krein [20], Birman-Krein [1], Yafaev [26] and the survey by Birman-Yafaev [2]. There is a huge litterature on the spectral shift function and we only quote the first two papers which are historical ones, the book [26] where one can also find proofs and a nice presentation on the subject, and the complete survey [2]. Notice that the essential spectrum of $A_{0}$, and hence the one of $A_{1}$, may be non empty; in particular, there can be absolutely continuous spectrum, therefore $s_{1}$ is an important tool in scattering theory. It is also called scattering phase because of the following Krein's formula

$$
\operatorname{Det}_{1} S(\lambda)=e^{2 i \pi s_{1}(\lambda)}, \quad \text { a.e. on } \sigma_{a c}\left(A_{0}\right),
$$


where $S(\lambda)$ is the scattering matrix of the pair $A_{0}, A_{1}$ at energy $\lambda$, and $\sigma_{a c}\left(A_{0}\right)$ the absolutely continuous spectrum of $A_{0}$. The function $s_{1}$ can be considered as a phase for another reason which is the following

$$
s_{1}(\lambda)=-\frac{1}{\pi} \lim _{\delta \searrow+0} \arg \operatorname{Det}_{1}\left(1+W\left(A_{0}-\lambda-i \delta\right)^{-1}\right), \quad \text { a.e. on } \mathbb{R} .
$$

In these last two formulas, we have used the functional Det ${ }_{1}$ which is defined as follows. The Schatten ideal $S_{1}$ of trace class operators is the set of bounded operators $T$ such that $|T|=\left(T^{\star} T\right)^{1 / 2}$ is compact with a spectrum in $l^{1}(\mathbb{N})$; if $T \in S_{1}$ then it is compact as well, its spectrum $\left(\lambda_{j}\right)_{j \in \mathbb{N}}$ is in $l^{1}(\mathbb{N})$ and one can set

$$
\operatorname{Det}_{1}(1+T)=\prod_{j \in \mathbb{N}}\left(1+\lambda_{j}\right)
$$

Formula (3) makes sense because, for trace class perturbations $W$, the operator $S(\lambda)-1$ is a trace class operator as well.

This perturbation theory by trace class operators will be called Birman-Krein's theory throughout this paper. We can justify the introduction of such concepts in a very elementary way. If $M$ is a $n \times n$ hermitian matrix $\left(M^{\star}=M\right)$, its spectrum $\Lambda_{1}, \cdots, \Lambda_{n}$ is real and given as the roots of its characteristic polynomial, or equivalently by the poles of $D(z)=\operatorname{Det}(M-z)^{-1}$. Using $\frac{d}{d z} \log D(z)=-\sum_{j=1}^{n}\left(\Lambda_{j}-z\right)^{-1}$, combined with the elementary identity $2 i \pi \delta_{0}(\lambda)=(\lambda-i 0)^{-1}-(\lambda+i 0)^{-1}$, and the fact that the imaginary part of $\log D(z)$ is the argument of $D(z)$, we get easily

$$
\lim _{\delta \searrow 0} \frac{d}{d \lambda} \arg D(\lambda+i \delta)=-\pi \sum_{j=1}^{n} \delta_{\Lambda_{j}}(\lambda)
$$

The right hand side, up to the factor $-\pi$, is the spectral measure associated to $M$, that is the derivative of the counting function $N(\lambda)$ defined as the number of eigenvalues of $M$ lower than $\lambda$. The connection with the Birman-Krein's theory is the following: since $\operatorname{Det}_{1}\left(1+W\left(A_{0}-z\right)^{-1}\right)=\operatorname{Det}_{1}\left(\left(A_{1}-z\right)\left(A_{0}-z\right)^{-1}\right)$, it can be viewed as " $\operatorname{Det}\left(A_{1}-z\right) / \operatorname{Det}\left(A_{0}-z\right)$ ", and formula (4) tells us that $s_{1}$ should be considered as the difference of the counting functions associated to $A_{0}$ and $A_{1}$ (this is compatible with formula (2)) and thus measures the shift of the spectrum of $A_{0}$ when we add the perturbation $W$. Of course this is completely heuristic since, in general, the spectra of $A_{0}$ and $A_{1}$ are not discrete, but it explains why the arguments of some determinants are interesting.

So far we have only considered operators $W$ which are trace class, but one can be interested in more general compact perturbations, for example with $W$ in the Hilbert-Schmidt class $S_{2}$. More generally, we now assume that $W \in S_{p}$, for some integer $p \geq 1$ (recall that $T \in S_{p}$ if $|T|$ is compact with a spectrum in $l^{p}(\mathbb{N})$ ); of course $S_{1} \varsubsetneqq S_{p}$ when $p \geq 2$, thus one cannot use Birman-Krein's theory. In order to replace (2), Koplienko introduced the following functional in [18]

$$
<u_{p}, f>=\operatorname{Tr}\left(f\left(A_{0}+W\right)-\sum_{j=0}^{p-1} \frac{1}{j !} \frac{d^{j}}{d \epsilon^{j}} f\left(A_{0}+\epsilon W\right)_{\mid \epsilon=0}\right) .
$$


In his paper, the functions $f$ are rational fractions; the point is that the operator considered on the right hand side of (6) is trace class. Heuristically this is clear because we get somehow "OO $\left(W^{p}\right)$ " which is trace class, by basic properties of the Schatten classes ( $W \in S_{p} \Rightarrow W^{p} \in S_{1}$ ). Analogously to formula (4), $u_{p}$ is naturally linked to the Fredholm determinant $\operatorname{Det}_{p}\left(1+W\left(A_{0}-z\right)^{-1}\right)$; we recall that if $T \in S_{p}$, its spectrum $\left(\lambda_{j}\right)_{j \in \mathbb{N}}$ belongs to $l^{p}(\mathbb{N})$ and one can define

$$
\operatorname{Det}_{p}(1+T)=\prod_{j \in \mathbb{N}}\left(1+\lambda_{j}\right) \exp \left(\sum_{k=1}^{p-1} \frac{(-1)^{k}}{k} \lambda_{j}^{k}\right)
$$

where the product is convergent since the Weierstrass function on the right hand side is $1+\mathcal{O}\left(\lambda_{j}^{p}\right)$. It is convinient to use such determinants (instead of $\operatorname{Det}_{1}\left(1+T^{p}\right)$ for example) because the multiplicity of $1+\lambda_{j}$ as a root of the product is independent of $p$ (for all $p$ such that the product exists).

This is the model of a perturbation theory that we may wish to apply to selfadjoint differential operators. However, for such operators, the perturbation is never compact, thus the application of the above considerations is not straightforward, except for the case $\rho>d$ (with the notation of (1)). This is the purpose of the next subsection.

\subsection{Differential operators}

Now we consider the differential operators $H_{0}$ and $H_{1}=H_{0}+V$ introduced in subsection 1.1. When $\rho>d$, the following difference is trace class

$$
\left(H_{1}+E\right)^{-N}-\left(H_{0}+E\right)^{-N}
$$

for some $E>0$ and $N>0$ large enough. This is due to the fact (with $p=1$ here) that

$$
\langle x\rangle^{-\nu}\langle D\rangle^{-\nu} \in S_{p} \quad \text { when } \nu>d / p .
$$

Using Birman's invariance principle (IP) (see [1]), we can use the theory of trace class perturbations of Birman-Krein to define a function $s_{1} \in L_{l o c}^{1}(\mathbb{R})$ such that

$$
\operatorname{Tr}\left(f\left(H_{1}\right)-f\left(H_{0}\right)\right)=-\int f^{\prime}(\lambda) s_{1}(\lambda) d \lambda, \quad f \in \mathcal{S}(\mathbb{R}) .
$$

To do so, we consider $A_{j}=\left(H_{j}+E\right)^{-N}, j=0,1$, and then

$$
s_{1}\left(H_{0}, H_{1}, \lambda\right)=-s_{1}\left(A_{0}, A_{1},(\lambda+E)^{-N}\right)
$$

satisfies (10). The function $s_{1}$ is uniquely by (10) up to a constant term. Moreover (10) shows clearly that $s_{1}$ is constant below the spectrum of $H_{1}$, thus we can normalize it with the condition $s_{1}(\lambda)=0$ when $\lambda \ll 0$. Moreover by Birman's IP, the scattering matrix of $H_{0}, H_{1}$ is still a trace class perturbation of identity and its determinant is $e^{2 i \pi s_{1}(\lambda)}$.

It is an important problem in scattering theory, for mathematical and physical reasons, to know the asymptotic behavior of $s_{1}$ when $\lambda \nearrow+\infty$. Two types of 
asymptotic expansions can be obtained, the weak and the strong (or pointwise) ones, and their applications are various: relative index theory, inverse problems, traces formulae, resonances...

The weak asymptotics are obtained by choosing a function $f$ in (10) which depends on a small parameter, and by considering the expansion with respect to this parameter. For example, we quote the so called heat expansion

$$
\operatorname{Tr}\left(e^{-t H_{1}}-e^{-t H_{0}}\right) \sim t^{-d / 2} \sum_{n \geq 0} \alpha_{n}^{1} t^{n}, \quad t \searrow+0
$$

which has been proved by Buslaev [11], Colin-De-Verdière [13], Guillopé [15], Robert [24] and Bruneau [8] in various settings. Such expansions can be obtained under more general conditions; in particular the unperturbed operator $H_{0}$ may have variable coefficients (periodic, for example) and no assumption on the classical flow is needed. This kind of expansion is similar to the one used to prove index theorems for elliptic operators on compact manifolds; actually (11) is a tool in the proof of relative index theorems see [3, 4, 8].

The pointwise asymptotic behavior of $s_{1}$ is much more difficult to study and depends strongly on the classical flow associated to $H_{1}$, i.e. the geodesics of the metric $g(x)=\left(g^{j k}(x)\right):=\left(g_{j k}(x)\right)^{-1}$. Notice that $g(x)-I_{d}=\mathcal{O}\left(\{x\}^{-\rho}\right)$, which means that the metric is asymptotically euclidean.

The existence of a (strong) complete asymptotic expansion can only be proved under a non trapping condition. The metric is non trapping if all the geodesics escape to infinity; equivalently this means that $\left|\Pi \Phi^{t}(x, \xi)\right| \rightarrow \infty$ when $|t| \rightarrow \infty$, uniformly on all compact subset of $T^{\star} \mathbb{R}^{d} \backslash 0$ (here $\Pi: T^{\star} \mathbb{R}^{d} \rightarrow \mathbb{R}^{d}$ is the projection and $\Phi^{t}$ is the Hamiltonian flow of the principal symbol of $H_{1}$ ). We quote the following theorem which states the result that we are going to generalize

Theorem 1.1 [12, 13, 15, 24, 25] Assume that $\rho>d$, then:

i) $s_{1}$ is smooth on $(0, \infty)$,

ii) if the metric is non trapping we have a complete asymptotic expansion

$$
s_{1}^{\prime}(\lambda) \sim \lambda^{\frac{d}{2}-1} \sum_{n \geq 0} \beta_{n}^{1} \lambda^{-n}, \quad \lambda \nearrow+\infty .
$$

This theorem was proved successively by Buslaev-Faddeev [12], Colin-De-Verdière [13], Guillopé [15] and Robert [24, 25]. We won't discuss the computation of the coefficients $\beta_{n}^{1}$ (and $\alpha_{n}^{1}$ ), but we just mention that $\beta_{1}^{n}$ is proportional to $\int_{\mathbb{R}^{d}}(\operatorname{det} g(x))^{1 / 2}-$ $1 d x$. Notice that the integral is convergent since $(\operatorname{det} g(x))^{1 / 2}-1=\mathcal{O}\left(\langle x\rangle^{-\rho}\right)$ with $\rho>d$.

Now we turn to the question that we want to address in this paper: what happens if we only know that $\rho>0$ ? By choosing $p \geq 1$ such that $\rho>d / p$, it is easy to prove that (8) belongs to the Schatten class $S_{p}$. Unfortunately, one cannot use Birman's IP any longer, if $p \geq 2$, to apply Koplienko's theory of $S_{p}$ perturbations, as mentionned in [18]. To overcome this problem, the following result, inspired by Koplienko's idea, was proved in $[5,6]$ 
Theorem 1.2 If $\rho>d / p$, then for all $f \in \mathcal{S}(\mathbb{R})$, we can define

$$
<u_{p}, f>=\operatorname{Tr}\left(f\left(H_{0}+V\right)-\sum_{j=0}^{p-1} \frac{1}{j !} \frac{d^{j}}{d \epsilon^{j}} f\left(H_{0}+\epsilon V\right)_{\left.\right|_{\epsilon=0}}\right),
$$

which means, in particular, that the operator considered on the right hand side is trace class.

The functional $u_{p}$ is a temperate distribution supported in $\operatorname{spec}\left(H_{1}\right)$, and the following heat expansion holds

$$
<u_{p}, e^{-t(.)}>\sim t^{-d / 2} \sum_{n \geq 0} \alpha_{n}^{p} t^{n}, \quad t \searrow+0,
$$

with the notation $e^{-t(.)}$ for the function $\lambda \mapsto e^{-t \lambda}$.

We must precise that in [19], Koplienko had already defined $u_{2}$ for the Schrödinger operator on the half-line. Moreover, the case $p=2$ is a special one in [18, 19], and was also studied for more general pseudo-differential operators (in any dimension) in $[5,6]$.

Another way of regularizing trace formulae for long range Schrödinger operators, still based on Taylor's formula, has been considered by Melin in [21] where weak expansions are proved.

\section{Results}

We want to consider two questions, the first one being the relation of $u_{p}$ with some scattering determinants. The second one deals with the asymptotic behavior of $u_{p}$. In this section $\rho>0$ is an arbitrary real number and $p \geq 1$ is an integer such that

$$
\rho>d / p
$$

As mentionned before, $V\left(H_{0}-z\right)^{-1}$ is not compact in general, unless

$\left(g_{j k}(x)\right)$ is the unit matrix, thus we cannot consider $\operatorname{Det}_{p}\left(1+V\left(H_{0}-z\right)^{-1}\right)$, and we have to extend the definition of the determinants. In order to extend this definition, we consider the following regularized Zeta function

$$
\zeta_{p}(z, s)=\operatorname{Tr}\left(\left(H_{0}+V-z\right)^{-s}-\sum_{j=0}^{p-1} \frac{1}{j !} \frac{d^{j}}{d \epsilon^{j}}\left(H_{0}+\epsilon V-z\right)_{\mid \epsilon=0}^{-s}\right)
$$

which is well defined for $z \in \mathbb{C} \backslash \mathbb{R}$ and $\operatorname{Re} s \gg 1$. When $p=1$, relations between Zeta functions and scattering determinants are reviewed and studied by Müller in [22].

For general $p \geq 1$, we prove the following theorem:

Theorem 2.1 [Y] i) The function $s \mapsto \zeta_{p}(z, s)$ has a meromorphic continuation to the complex plane which is regular at $s=0$.

ii) The function $D_{p}(z)=\exp \left(-\partial_{s} \zeta_{p}(z, 0)\right)$ is holomorphic on $\mathbb{C} \backslash \mathbb{R}$ and we have

$$
\lim _{\delta \searrow+0} \frac{d}{d \lambda} \arg D_{p}(\lambda+i \delta)=-\pi u_{p}(\lambda), \quad \text { in } \mathcal{D}^{\prime}(\mathbb{R}) .
$$


The only non elementary tool of the proof is the heat expansion (12); most of the other ingredients can be found in [22].

By analogy with the results for $p=1$ we can set:

Definition 2.2 i) $D_{p}(z)$ is the regularized determinant of order $p$ associated to $H_{0}, H_{1}$.

ii) The regularized scattering phase $s_{p} \in \mathcal{S}^{\prime}(\mathbb{R})$ is the unique primitive of $u_{p}$ which vanishes near $-\infty$.

We can prove that $D_{p}(z)$ coincides with the Fredholm determinant, for a lot of relatively compact perturbations. For example, if $V$ is only a potential which is $\mathcal{O}\left(\langle x\rangle^{-\rho}\right)$, and if $2>d / p$, then $V\left(H_{0}-z\right)^{-1} \in S_{p}$ (by (9)) and $D_{p}(z)=\operatorname{Det}_{p}(1+$ $\left.V\left(H_{0}-z\right)^{-1}\right)$, with $\operatorname{Det}_{p}$ defined by $(7)$. This justify the name determinant for $D_{p}(z)$.

Notice moreover that the definition of $s_{p}$ makes sense since $u_{p}$ is a temperate distribution which vanishes below the spectrum of $H_{1}$. Of course, when $p=1$, we get the usual SSF, and (13) can be considered as a generalization of (4).

Now we are in position to state our main result.

Theorem 2.3 Here we suppose that $d \geq 2$. Assume that $\rho>d / p$, then:

i) $s_{p}$ is smooth on $(0, \infty) \backslash \sigma_{p p}\left(H_{1}\right)$,

ii) if the metric is non trapping we have a complete asymptotic expansion

$$
s_{p}^{\prime}(\lambda) \sim \lambda^{\frac{d}{2}-1} \sum_{n \geq 0} \beta_{n}^{p} \lambda^{-n}, \quad \lambda \nearrow+\infty
$$

A similar result concerning perturbations by potentials (hence non-trapping) has been announced by Hitrik-Polterovitch [16], where Melin's regularization [21] is considered.

We conclude with remarks on other asymptotic properties of $s_{p}$ (see [7]), when trapped trajectories exist. We can prove a Weyl formula, $s_{p}(\lambda)=c \lambda^{d / 2}+\mathcal{O}\left(\lambda^{\frac{d-1}{2}}\right)$, however our proof relies on a priori estimates for $\left(H_{0}+V-\lambda \pm i 0\right)^{-1}$ of exponential type. Such estimates are known for a wide class of long range perturbations, thanks to the works of Burq [10] and Bruneau-Petkov [9]. Nevertheless, we recall that, for $p=1$ such estimates are not necessary; in [24], Robert proves the Weyl formula without any assumption on the resolvent, by splitting $s_{1}$ into non deacreasing terms which allows him to use a Tauberian theorem. For general $p$, our method is different.

In [7], we also prove a Breit-Wigner formula for $s_{p}$, in the semiclassical limit: it describes how $s_{p}^{\prime}$ can blow up near trapping energy levels, when resonances are close to the real axis.

\section{Proof of theorem 2.3}

We are going to prove the existence of the asymptotic expansion, since the arguments of the proof of the smoothness are contained in what follows.

By a rescaling argument, the problem can be treated as a semiclassical one by considering the operators $\hat{H}_{\epsilon}:=h^{2}\left(H_{0}+\epsilon V\right)$, with the small parameter $h=\lambda^{-1 / 2}$. 
This is a consequence of the simple fact that $u_{p}\left(\mu / h^{2}\right)=h^{2} u_{p}(\mu, h)$, if $u_{p}(., h)$ is associated to $\hat{H}_{0}, \hat{H}_{1}$. Thus we are going to prove an expansion with respect to $h \searrow 0$ for $u_{p}(\mu, h)$, when the rescaled energy variable $\mu$ is close to 1 .

We assume first that the coefficients of $V$ are compactly supported. More precisely, we consider a sequence $V_{n}$ converging to $V$ in the sense that the compactly supported coefficients of $V_{n}$ converge to the ones of $V$ in $B_{\rho^{\prime}}$ for all $\rho^{\prime}<\rho$, where

$$
a \in B_{\rho^{\prime}} \Leftrightarrow \sup _{\mathbb{R}^{d}}\left|\langle x\rangle^{\rho^{\prime}+|\alpha|} \partial^{\alpha} a(x)\right|<\infty, \quad \forall \alpha .
$$

Notice that, if $\rho>d / p$, we can choose $\rho>\rho^{\prime}>d / p$.

Then, we can define $u_{p}^{n}$ (associated to $\hat{H}_{0}, \hat{H}_{0}+\hat{V}_{n}$ ) for all $p \geq 1$ and by considering $u_{1}^{n}(\mu, \epsilon, h)$, associated to $\hat{H}_{0}, \hat{H}_{0}+\epsilon \hat{V}_{n}$, we have

$$
u_{p}^{n}(\mu, h)=u_{1}^{n}(\mu, 1, h)-\sum_{j=0}^{p-1} \frac{1}{j !} \frac{\partial^{j}}{\partial \epsilon^{j}} u_{1}^{n}(\mu, 0, h)
$$

in the weak sense, i.e. the equality makes sense when tested against a Schwartz's function in the $\mu$ variable. The assumption on the fast decay of the coefficients of $V_{n}$ is only needed to use $u_{1}$. Moreover, in the weak sense again, we have near $\mu=1$

$$
u_{1}^{n}(\mu, \epsilon, h)=\operatorname{Tr}\left(f\left(\hat{H}_{\epsilon}^{n}\right) \frac{\partial E_{\epsilon}^{n}}{\partial \mu}-f\left(\hat{H}_{0}\right) \frac{\partial E_{0}}{\partial \mu}\right), \quad \hat{H}_{\epsilon}^{n}=\hat{H}_{0}+\epsilon \hat{V}_{n}
$$

where $E_{\epsilon}^{n}($.$) is the spectral resolution associated to \hat{H}_{\epsilon}^{n}$, and $f \in C_{0}^{\infty}(\mathbb{R})$ such that $f \equiv 1$ near $\mu=1$. Formulas (14) and (15) suggests that we have to study the differentiability of the spectral projections with respect to $\epsilon$, or equivalently, after semiclassical Fourier transform, the one of the propagators $U_{\epsilon}^{n}(t)=\exp \left(-i t \hat{H}_{\epsilon}^{n} / h\right)$. To do so, we are going to use Isozaki-Kitada's parametrix (see [17] and [14, 25]).

Notice that, by simple considerations on functional calculus (see [5, 6]), we have $u_{p}^{n} \rightarrow u_{p}$ in $\mathcal{D}^{\prime}\left(f^{-1}(1)\right)$. The point here is to prove that the limit of $u_{p}^{n}$ belongs to $C^{\infty}$ and has an expansion in increasing powers of $h$ (with respect to the $C^{\infty}$ topology).

We choose a cutoff function $\chi \in C_{0}^{\infty}$, with $\chi \equiv 1$ on the ball of radius $R>0$ (we shall choose $R$ later).

First we consider $\operatorname{Tr}\left(\chi f\left(\hat{H}_{1}^{n}\right) d E_{1}^{n} / d \mu\right)$. Thanks to $\chi$ we can let $n$ go to infinity. Moreover, the existence of an asymptotic expansion for $\operatorname{Tr}\left(\chi f\left(\hat{H}_{1}\right) d E_{1} / d \mu\right)$ is already well known (see [23]) under the non trapping condition.

Then it is not very difficult to prove that

$$
\frac{\partial^{j}}{\partial \epsilon^{j}} \operatorname{Tr}\left(f\left(\hat{H}_{\epsilon}\right) \frac{\partial E_{\epsilon}}{\partial \mu} \chi\right)_{\mid \epsilon=0}
$$

has a complete asymptotic expansion. Notice that, here again, it is sufficient to consider directly the limit $n \rightarrow \infty$ itself. We consider its semiclassical Fourier transform, swhich is a linear combination of the following type of functions:

$$
h^{-k} \int_{0}^{t} \int_{0}^{t_{1}} \cdots \int_{0}^{t_{k-1}} \operatorname{Tr}\left(\mathcal{K}_{k}\left(t-t_{1}, t_{1}-t_{2}, \cdots, t_{k-1}-t_{k}, t_{k}, h\right) \chi\right) d t_{k} \cdots d t_{2} d t_{1},
$$


where $0 \leq k \leq j$, and by convention, there is no $\int \operatorname{sign}$ if $k=0$, and $t_{0}=t$. The operator $\mathcal{K}_{k}\left(t_{1}, \cdots, t_{k+1}, h\right)$ has the following form

$$
\mathcal{K}_{k}\left(t_{1}, \cdots, t_{k+1}, h\right)=a_{1}(x, h D, h) U_{0}\left(t_{1}\right) a_{2}(x, h D, h) U_{0}\left(t_{2}\right) \cdots a_{k+1}(x, h D, h) U_{0}\left(t_{k+1}\right)
$$

This is simply due to the fact that $f\left(\hat{H}_{\epsilon}\right)$ is a smooth family of $h$-pseudo-differential operators, with respect to $\epsilon \in[0,1]$ and to the formula

$$
\frac{\partial}{\partial \epsilon} U_{\epsilon}(t)=-\frac{i}{h} \int_{0}^{t} U_{\epsilon}\left(t-t_{1}\right) \hat{V} U_{\epsilon}\left(t_{1}\right) d t_{1}, \quad \hat{V}=h^{2} V .
$$

By looking at the explicit Schwartz kernel of $\mathcal{K}_{k}$, we can prove that the trace in (16) is $\mathcal{O}\left((h / t)^{\infty}\right)$ when $|t|>T$ (for arbitrary $T>0$ ), by a non stationary phase argument. Then we get the expected complete asymptotic expansion thanks to the stationary phase theorem, by computing the inverse Fourier transform of (16) multiplied by a time cutoff.

We are now left to the study of $\operatorname{Tr}\left(\left(f\left(\hat{H}_{\epsilon}^{n}\right) d E_{\epsilon}^{n} / d \mu-f\left(\hat{H}_{0}\right) d E_{0} / d \mu\right)(1-\chi)\right)$ which we are going to analyze again thanks to its semiclassical Fourier transform. Thanks to the energy cutoff $f$, and to the factor $1-\chi$, it is rather simple to prove that we only have to consider

$$
\begin{gathered}
\operatorname{Tr}\left(\left(U_{\epsilon}^{n}(t)-U_{0}(t)\right) \chi_{ \pm}(x, h D)\right) \\
\operatorname{Tr}\left(U_{\epsilon}^{n}(t) \tilde{\chi}_{ \pm}(x, h D)\left(f\left(\hat{H}_{\epsilon}^{n}\right)-f\left(\hat{H}_{0}\right)\right)\right)
\end{gathered}
$$

where $\chi_{ \pm}, \tilde{\chi}_{ \pm}$are symbols supported in the following type of areas

$$
\Gamma^{ \pm}\left(R, J, \sigma_{ \pm}\right)=\left\{\left.(x, \xi) \in \mathbb{R}^{2 d}|| x|>R,| \xi\right|^{2} \in J,\langle x, \xi\rangle>\sigma_{ \pm}|x||\xi|\right\}
$$

where $J$ is a neighborhood of 1 . In (18) we have $\chi_{+}(x, \xi)+\chi_{-}(x, \xi)=(1-\chi)(x) f\left(\xi^{2}\right)$, and in $(19), \tilde{\chi}_{+}(x, \xi)+\tilde{\chi}_{-}(x, \xi)=(1-\chi)(x) \tilde{f}\left(\xi^{2}\right)$, for some $\tilde{f}$ such that $\tilde{f} f=f$. We can use such a decomposition because on the support of the principal symbol of $(1-\chi) f\left(\hat{H}_{\epsilon}^{n}\right)$, which is $(1-\chi)(x) f\left(\xi^{2}+\mathcal{O}\left(\langle x\rangle^{-\rho}\right)\right), \xi^{2}$ must stay near the support of $f$ since $x$ is large.

This microlocal partition of unit is interesting because now, we can use the following Isozaki-Kitada's expansion: provided that $R$ is large enough, we have for all $N \geq 0$

$$
U_{\epsilon}^{n}(t) \chi_{ \pm}(x, h D)=A_{N}^{n \pm}(h, \epsilon) U_{0}(t) B_{N}^{n \pm}(h, \epsilon)^{\star}+h^{N} R_{N}^{n \pm}(h, \epsilon, t), \quad \pm t \geq 0 .
$$

This means that (20) is valid for $t \geq 0$ in the + case, and $t \leq 0$ for - . The operators $A_{N}^{n \pm}(h, \epsilon)$ and $B_{N}^{n \pm}(h, \epsilon)$ are Fourier integral operators (FIO) associated to time independent phase functions $\varphi_{n \pm}$; for example we have $A_{N}^{n \pm}(h, \epsilon)=$ $\sum_{k \leq N} h^{k} J_{\varphi_{ \pm}}\left(a_{k}^{n \pm}\right)$ where

$$
J_{\varphi_{ \pm}}\left(a_{k}^{n \pm}\right) u(x)=(2 \pi h)^{-d} \iint e^{i\left(\varphi_{ \pm}^{n}(x, \xi, \epsilon, h)-\langle y, \xi\rangle\right) / h} a_{k}^{n \pm}(x, \xi, \epsilon) u(y) d y d \xi
$$


We improve the estimates on the phase functions and the symbols $a_{k}^{n \pm}$ that are established in $[17,14,25]$ by considering the dependence with respect to the parameter $\epsilon$, and also with respect to $n$. More precisely, we prove in [7] that

$$
\begin{aligned}
\partial_{x}^{\alpha} \partial_{\xi}^{\beta} \partial_{\epsilon}^{j} \partial_{h}^{l}\left(\varphi_{ \pm}^{n}(x, \xi, \epsilon, h)-\langle x, \xi\rangle\right) & =\mathcal{O}\left(\langle x\rangle^{1-|\alpha|-\rho^{\prime} j_{+}}\right), \quad j_{+}=\sup (j, 1)(21) \\
\partial_{x}^{\alpha} \partial_{\xi}^{\beta} \partial_{\epsilon}^{j} \partial_{h}^{l}\left(a_{0}^{n \pm}(x, \xi, \epsilon)-1\right) & =\mathcal{O}\left(\langle x\rangle^{-|\alpha|-\rho^{\prime} j_{+}}\langle\xi\rangle^{-\infty}\right), \quad k \geq 1 \\
\partial_{x}^{\alpha} \partial_{\xi}^{\beta} \partial_{\epsilon}^{j} \partial_{h}^{n} a_{k}^{n \pm}(x, \xi, \epsilon) & =\mathcal{O}\left(\langle x\rangle^{-k-|\alpha|-\rho^{\prime} j_{+}}\langle\xi\rangle^{-\infty}\right), \quad
\end{aligned}
$$

(There are similar estimates for the symbols of $B_{N}^{n \pm}(h, \epsilon)$.) Moreover we can control all these estimates with a finite number of semi-norms of the coefficients of $V_{n}$ in $B_{\rho^{\prime}}$. Precisely this means that the semi-norms of $\varphi_{ \pm}^{n}-\varphi_{ \pm}^{n^{\prime}}$ and $a_{k}^{n \pm}-a_{k}^{n^{\prime}}{ }^{ \pm}, k \geq 0$, can be estimated by semi-norms of the coefficients of $V_{n}-V_{n^{\prime}}$ in $B_{\rho^{\prime}}$.

Let's mention finally that the symbols are chosen so that

$$
B_{N}^{n \pm}(h, \epsilon)^{\star} A_{N}^{n \pm}(h, \epsilon)=\chi_{ \pm}(x, h D)+\mathcal{O}\left(h^{N / 2}\right)
$$

where the remainder is a trace class operator.

Now we can come back to the study of (18) and (19), taking (20) into account. We forget about the terms $R_{N}^{n}{ }^{ \pm}(h, \epsilon, t)$. They can be treated as in [25], using propagation estimates on $U_{1}(t)$ (thanks to the non trapping condition) when $\epsilon=$ 1 , and with the same non stationary phase argument as before, concerning their derivatives at $\epsilon=0$.

By standard argument (see [23]), we just have to consider the case + for $t \geq 0$. Moreover, by the cyclicity trick of Robert [25], we have

$$
\begin{gathered}
\operatorname{Tr}\left(A_{N}^{n+}(h, \epsilon) U_{0}(t) B_{N}^{n+}(h, \epsilon)^{\star}-B_{N}^{n+}(h, 0)^{\star} A_{N}^{n+}(h, 0) U_{0}(t)\right) \\
=\operatorname{Tr}\left(\left(B_{N}^{n+}(h, \epsilon)^{\star} A_{N}^{n+}(h, \epsilon)-B_{N}^{n+}(h, 0)^{\star} A_{N}^{n+}(h, 0)\right) U_{0}(t)\right) \\
=\operatorname{Tr}\left(\mathcal{O}\left(h^{N / 2}\right) U_{0}(t)\right)
\end{gathered}
$$

thanks to (24), thus (18) doesn't contribute.

Now we have to study (19) that we can write, up to a remainder $\mathcal{O}\left(h^{N}\right)$,

$$
\operatorname{Tr}\left(U_{0}(t) \tilde{B}_{N}^{n \pm}(h, \epsilon)^{\star} \kappa^{n}(x, h D, h, \epsilon) \tilde{A}_{N}^{n \pm}(h, \epsilon)\right)
$$

since $f\left(\hat{H}_{\epsilon}^{n}\right)-f\left(\hat{H}_{0}\right)$ is pseudodifferential. Here $\tilde{A}_{N}^{n \pm}$ and $\tilde{B}_{N}^{n \pm}$ are FIO as in (20), associated to $\tilde{\chi}_{ \pm}$.

Then using the estimates (21), (22), (23) and the semiclassical Egorov's theorem, it is very easy to prove that $\mathcal{A}^{n \pm}(h, \epsilon):=\tilde{B}_{N}^{n}{ }^{ \pm}(h, \epsilon)^{\star} \kappa^{n}(x, h D, h, \epsilon) \tilde{A}_{N}^{n}{ }^{ \pm}(h, \epsilon)$ satisfies

$$
\mathcal{A}^{n \pm}(h, 1)-\sum_{j=0}^{p-1} \frac{1}{j !} \frac{d^{j}}{d \epsilon^{j}} \mathcal{A}^{n \pm}(h, \epsilon)_{\left.\right|_{\epsilon=0}}=c_{N}^{n \pm}(x, h D, h)
$$

where the symbols $c_{N}^{n \pm} \in S^{-\infty,-p \rho^{\prime}}$ depend continuously on the symbols of $V_{n}$ in $B_{\rho^{\prime}}$. The class of symbols $S^{-\infty,-p \rho^{\prime}}$ is the following

$$
m \in S^{-\infty,-p \rho^{\prime}} \Leftrightarrow \partial_{x}^{\alpha} \partial_{\xi}^{\beta} m(x, \xi)=\mathcal{O}\left(\langle\xi\rangle^{-\infty}\langle x\rangle^{-p \rho^{\prime}}\right), \forall \alpha, \beta
$$


and the point is that all the corresponding operators are trace class, since $p \rho^{\prime}>d$. Thus we can let $n \rightarrow \infty$, which yields $c^{n \pm}(x, h D, h) \rightarrow c^{ \pm}(x, h D, h)$ in the trace class. Then the asymptotic expansion of $\int_{ \pm t \geq 0} e^{i \mu t / h} \operatorname{Tr}\left(c_{N}^{ \pm}(x, h D, h) U_{0}(t)\right) d t$ is a simple consequence of the stationary phase theorem, because we can give the explicit kernel of the operator and then express the trace as an oscillatory integral (see [25]). The theorem is proved.

\section{References}

[1] M. Sh. Birman, M. G. Krein, On the theory of wave operators and scattering operators, Dokl. Akad. Nauk SSSR, 144, 475-478 (1962).

[2] M. Sh. Birman, D. R. YAfaev, The spectral shift function. The work of M. G. Krein and its further development, St. Petersburg Math. J. 4, 833-870 (1993).

[3] D. Bollé, F. Gesztesy, H. Grosse, W. Schweiger, B. Simon, Witten index, axial anomaly and Krein's spectral function in supersymmetric quantum mechanics, J. Math. Phys. 28, 1512-1525 (1987).

[4] N. Borisov, W. Müller, R. Schrader, Relative index, axial anomaly and Krein's spectral shift function in supersymmetric scattering theory, Commun. Math. Phys. 114, 475-513 (1988).

[5] J.M. Bouclet, Distributions spectrales pour des opérateurs perturbés, $\mathrm{PhD}$ thesis, Nantes University (2000).

[6] —, Traces formulae for relatively Hilbert-Schmidt perturbations, Asymptotic Analysis, to appear.

[7] Spectral distributions for long range perturbations, preprint of Sussex University, (2002).

[8] V. Bruneau, Propriétés asymptotiques du spectre continu d'opérateurs de Dirac, PhD Thesis, University of Nantes, (1995).

[9] V. Bruneau, V. Petkov, Semiclassical estimates for trapping perturbations, Commun. Math. Phys. 213, No. 2, 413-432 (2000).

[10] N. BuRQ, Lower bounds for shape resonances widths of long range Schrödinger operators, American Journal of Math, to appear.

[11] V. S. Buslaev, Trace formulas and some asymptotic estimates of the resolvent kernel for the Schrödinger equation in three dimensional space, Problemy Mat. Fiz. 1, 82-101 (1966).

[12] V. S. Buslaev, L. D. Faddeev, Formulas for traces for a singular SturmLiouville differential operator, Sov. Math. Dokl. 1, 451-454 (1960). 
[13] Y. COLIN-DE-Verdière, Une formule de trace pour l'opérateur de Schrödinger dans $\mathbb{R}^{3}$, Ann. Sci. E.N.S., IV sér. 14, 27-39 (1981).

[14] C. GÉrard, A. Martinez , Prolongement méromorphe de la matrice de scattering pour des problèmes à deux corps à longue portée, Ann. I.H.P., Phys. Théor. 51, No.1, 81-110, (1989).

[15] L. Guillopé, Une formule de trace pour l'opérateur de Schrödinger dans $\mathbb{R}^{n}$, thèse de doctorat, Université de Grenoble (1981).

[16] M. Hitrik, I. Polterovich , Regularized traces and Taylor expansions for the heat semigroup, preprint, (2001).

[17] H. Isozaki, H. KitadA , Modified wave operators with time independant modifiers, J. Fac. Sci., Univ. Tokyo, Sect. I A 32, 77-104 (1985).

[18] L.S. Koplienko, Trace formula for non trace-class perturbations, Sib. Math. J. 25, 735-743 (1984).

[19] _ Regularized spectral shift function for one dimensional Schrödinger operator with slowly decreasing potential, Sib. Math. J. 26, 365-369 (1985).

[20] M. G. KReIn, Perturbation determinants and a formula for the trace of unitary and selfadjoint operators, Soviet Math. Dokl. 3, 707-710 (1962).

[21] A. MeLin, Trace distributions associated to the Schrödinger operator, J. Anal. Math. 59, 133-160 (1992).

[22] W. MüLLER, Relative zeta functions, relative determinants and scattering theory, Commun. Math. Phys. 192 (2), 309-347 (1998).

[23] D. RoBERT, On long range scattering for perturbations of Laplace operators, J. Anal. Math. 59, 189-203 (1992).

[24] _ Asymptotique de la phase de diffusion à haute énergie pour des perturbations du second ordre du Laplacien, Ann. Sci. École Norm. Sup., 4ème série, No. 25, 107-134 (1992).

[25] _ Relative time delay for perturbations of elliptic operators and semiclassical asymptotics, J. Funct. Anal. 126, No.1, 36-82 (1994).

[26] D. YAfaev, Mathematical scattering theory. General theory, vol. 105, A.M.S. Rhode Island (1992).

UNIVERSITY OF SUSSEX,

FAlmer, Brighton BN1 9QH, UK

j.m.bouclet@sussex.ac.uk 